\title{
JAK2 Protein Variant
}

National Cancer Institute

\section{Source}

National Cancer Institute. JAK2 Protein Variant. NCI Thesaurus. Code C105906.

A variation in the amino acid sequence for the tyrosine-protein kinase JAK2 protein. 